\title{
Dyskinesia in a Child: A Concern for a Rare Neuro-Metabolic Disorder
}

\author{
Ayesha Hafeez, Safia Fatima, Nayyar Chaudhry and Muhammad Tahir Khadim
}

\begin{abstract}
A 3-year child is discussed who presented with dyskinesia, large head size, developmental delay, and recurrent infections necessitating multiple hospital admissions. The diagnosis was not made at initial presentation or even after multiple hospital admissions. An organic acidemia was suspected, based on raised ammonia and lactate levels and metabolic acidosis and the diagnosis of glutaric aciduria Type 1 was established by finding markedly elevated levels of glutaric acid and its specific metabolites on urine organic acids analysis by gas chromatography-mass spectrometry, in the setting of specific clinical features. Further supporting evidence was provided by CT scan brain showing subdural hygroma along left cerebral hemisphere causing gyral flattening and widening of sylvian fissure.
\end{abstract}

Key Words: Inherited metabolic disorder, Organic acidemia, Glutaric aciduria type I.

\section{INTRODUCTION}

Glutaric aciduria (GA), type 1 , is an inherited metabolic disorder (IMD) due to deficiency of glutaryl-co A dehydrogenase $(\mathrm{GCDH})$ enzyme that breaks down lysine, hydroxylysine and tryptophan. This results in elevated glutaric acid and other metabolites, 3-hydroxyglutaric acid, glutaconic acid, and glutarylcarnitine, accumulation of which causes damage to the brain, particularly the basal ganglia, which regulate movement. The neurological symptoms include spasticity, dystonia, developmental delay, seizures, feeding difficulties, swelling of brain, encephalopathic coma, or even death. ${ }^{1}$ It can occur in all races and ethnicities with a worldwide estimated incidence of 1 in 100,000 . Individuals with this condition have low life expectancy with $10 \%$ deaths in first decade and more than half cannot survive beyond 35 years of age. Two types of screening modes are used worldwide; general screening with dry blood spot of infant by screening acylcarnitine on tandem mass spectrometry and selected screening on urine organic acids by gas chromatography mass spectrometry (GCMS). High risk cases, whose urine organic acids show significant results, can further be analysed and confirmed by mutation analysis of GCDH or enzyme level detection. Proper counselling of parents should be done by informing them in detail about the natural history and the particular neurological sequelae of GA type I. They should be advised about the nutritional management for preventing the complications and

Department of Chemical Pathology and Endocrinology, AFIP, National University of Medical Sciences, Rawalpindi, Pakistan

Correspondence: Dr. Ayesha Hafeez, Department of Chemical Pathology and Endocrinology, AFIP, National University of

Medical Sciences, Rawalpindi, Pakistan

E-mail: ayeshahafeez30@yahoo.com

Received: May 10, 2018; Accepted: August 08, 2018 emergency treatment in case of encephalopathy. Metabolic treatments include a low lysine diet, carnitine supplementation, and intensified emergency treatment during acute episodes of inter-current illness. ${ }^{2}$

If treated early and diligently, by an experienced interdisciplinary team, many of these children can lead a healthy life with normal or near normal neuropsychiatric development. ${ }^{3}$ Children who have undergone a metabolic crisis are more prone to develop permanent disability, i.e. severe motor difficulties and involuntary spastic movements.

\section{CASE REPORT}

A 3-year male child, resident of Swat, was referred to the Pediatric Metabolic Department of Armed Forced Institute of Pathology (AFIP), Rawalpindi, for evaluation of a suspected IMD. He was brought by his parents, with complaints of impairment of voluntary movements, and inability to maintain posture while walking for the past 6 months. The parents had also noticed a progressive increase in head size over the last two years, accompanied with delayed developmental milestones. There was history of multiple hospital admissions due to recurrent respiratory tract infections over the past one and a half year. There was no history of seizures, loss of consciousness, urinary or fecal incontinence, fever, headache or trauma, and no significant past surgical history. He had a full-term, uneventful, delivery at home, was breast fed for two years and weaning started at 6 months of age. His developmental milestones were delayed as he started neck holding at 7 months, sitting at 1 year and walking at $2 \frac{1}{2} \mathrm{yrs}$. of age. His parents had non-consanguineous marriage. His eldest brother died at age of 8 years due to a probable cerebrovascular accident with a strong suspicion of some IMD, which could not be diagnosed at that time. On general physical examination, the child was afebrile with a heart rate of 


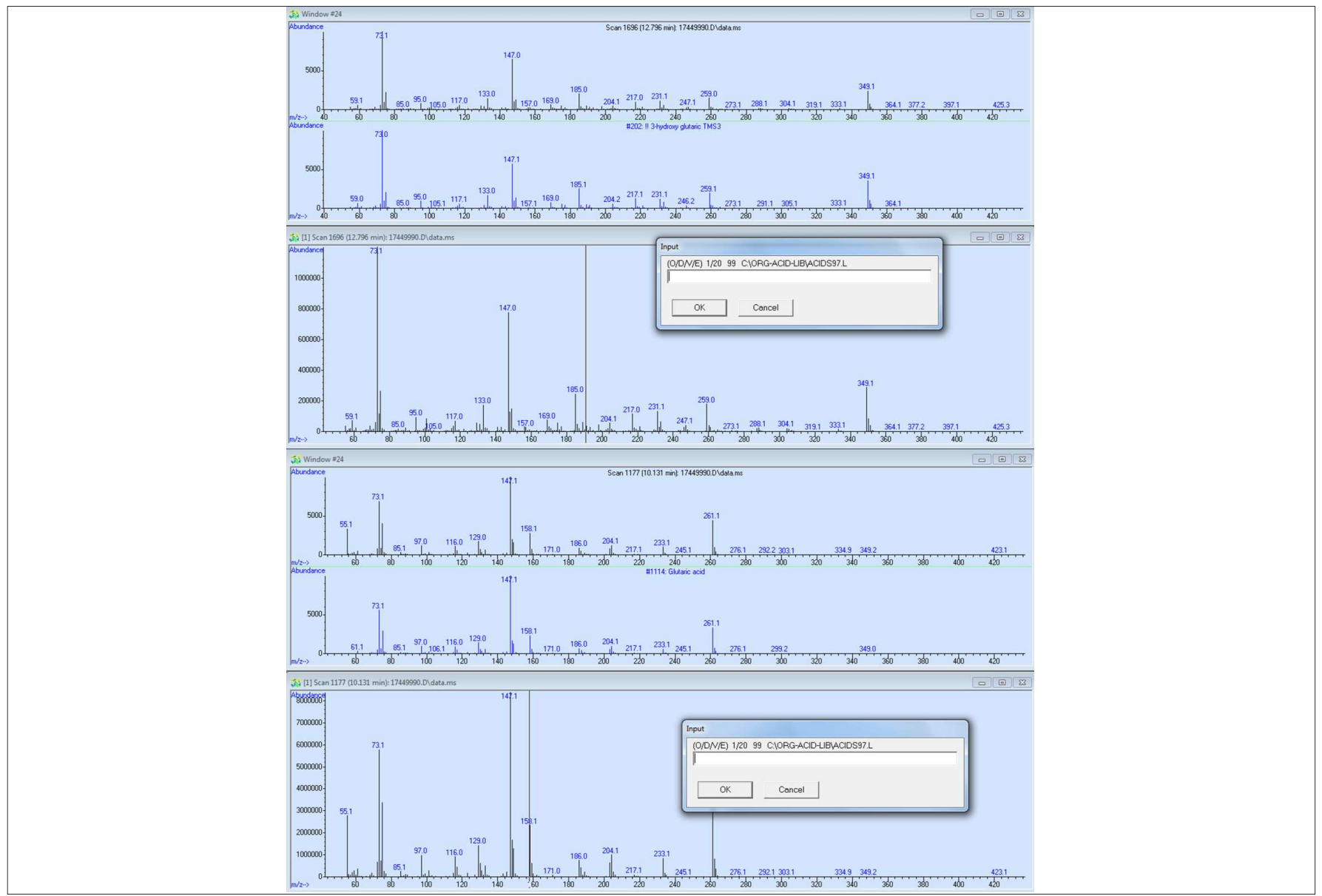

Figure 1: Gas chromotography- Mass spectrometry chromatogram showing significant peaks in the urine for glutaric acid, 3-hydroxy glutaric acid and 4 hydroxy phenylacetic acids.

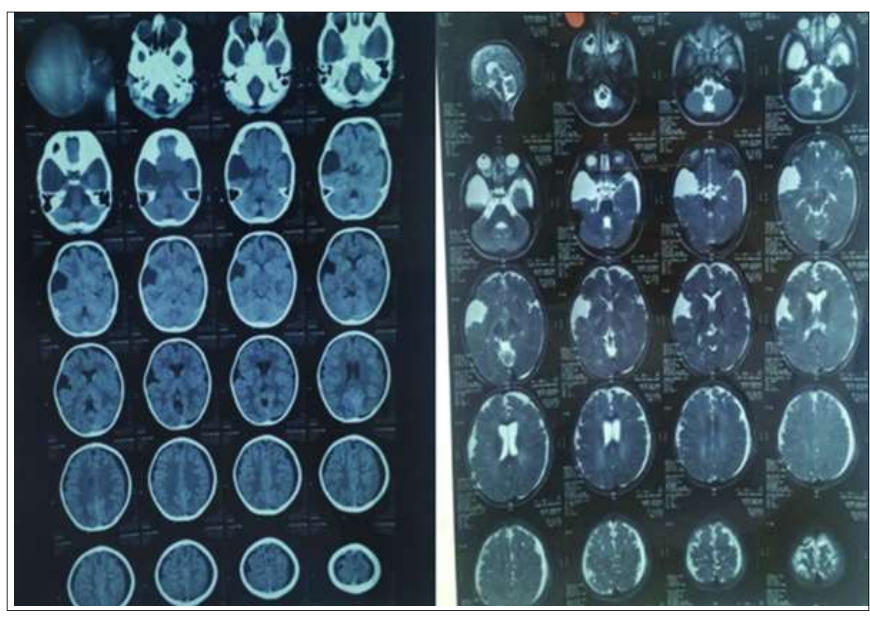

Figure 2: Showing CT scan head without contrast and MRI brain of a child presenting with dyskinesia.

90/min (regular), blood pressure of $110 / 75 \mathrm{~mm} \mathrm{Hg}$ and respiratory rate of $21 / \mathrm{min}$. His body weight was $13 \mathrm{Kg}$ $\left(<25^{\text {th }}\right.$ percentile); height was $52 \mathrm{~cm}\left(<5^{\text {th }}\right.$ percentile), while rest of the general physical examination was unremarkable. Systemic examination did not reveal any significant abnormality except central nervous system showing large head size with anterior fontanelle closure. His muscle tone was normal and plantars were downgoing. On laboratory investigations, his complete blood counts, plasma glucose, renal function tests, and liver function tests were unremarkable. Plasma ammonia levels were raised upto $135.8 \mu \mathrm{moll} / \mathrm{L}$ (range: 12-47), and so was the plasma lactate $3.0 \mathrm{mmol} / \mathrm{L}$; range $=0.5-2.22$ ). Arterial blood gas analysis showed metabolic acidosis. Urine for ketone bodies, nonglucose reducing substances and qualitative metabolic screening were negative. The quantitative plasma amino acid profile by Ion Exchange Chromatography, on Biochrome 30+ amino acid analyser was unremarkable. Urine organic acids analysis was done on GCMS and the chromatogram revealed marked elevation of glutaric acid, (Reference value $<5 \mu \mathrm{mol} / \mathrm{mmol}$ of creatinine ${ }^{5}$ ), 3 hydroxy glutaric acid (Reference value $<8 \mu \mathrm{mol} / \mathrm{mmol}$ of creatinine ${ }^{5}$ ) along with phenylacetic acids (Reference value $<10 \mu \mathrm{mol} / \mathrm{mmol}$ of creatinine) ${ }^{5}$ (Figure 1 ). CT scan brain showed findings of subdural hygroma along left cerebral hemisphere causing gyral flattening and widening of sylvian fissure while MRI brain showed a large right temporal fossa arachnoid cyst of approximately $70 \times 42 \mathrm{~mm}$ size with symmetrical abnormal T2WS/FLAIR hyper-intense areas in periventricular deep white matter bilaterally, suggestive of leukodystrophy (Figure 2). Based on clinical presentation, initial biochemical 
screening, plasma amino acids, urine organic acid analysis and radiological findings, the case was labelled as GA, type 1. Enzymatic diagnosis could not be achieved due to the lack of diagnostic facility in this set-up. His parents were counselled about the disease, its life expectancy, and the neural complications associated with it. As there is no specific treatment available, parents were advised to give him low protein and low lysine diet alongwith carnitine nutritional supplementation. They were further advised to ensure avoidance of prolonged fasting intervals for the child and to be particularly cautious during any acute illness, to prevent any further central nervous system complication. He will be followed on six-monthly basis for checking any improvement in his biochemical profile and mental activity with diet modification.

\section{DISCUSSION}

GA, type 1, mostly due to GCGD deficiency, is an intoxication group IMD, with neurological symptoms being a prominent feature. The clue to diagnosis in the case presented was macrocephaly with central nervous system (CNS) abnormalities manifested as dyskinesia; and the diagnosis was supported by biochemical evidence of elevated ammonia and lactate and increased excretion of glutaric acids and its metabolites on urine organic acids screening by GC-MS,4,5 along with supported evidence by gross anatomical abnormalities of CNS on CT-Scan Brain. In the presence of clinical features supported by biochemical findings, especially those of organic acid screening, a need for further supportive confirmation by the more cumbersome acylcarnitine analysis was not felt, as this GC-MS pattern of organic acids observed in urine samples from individuals with GCDH is not seen in any other metabolic disorder and is quite typical for GA, type 1, as supported by literature. 6 Various studies have supported the fact that GA, type 1 should be strongly suspected in a child with macrocephaly and dyskinesia, together with progressive atrophic changes on neuro-imaging studies. 7,8 The history of death of his sibling at eight years of age consequent to a probable cerebrovascular accident, further strengthened the suspicion of a neurometabolic disorder indicating that genetics has a strong role to play in this disorder. Various studies have supported this evidence. 9

Deficiency of GCDH leads to brain-basal ganglia accumulation of lysine, hydroxylysine, and trptophan, with glutaric acids and its subtypes. These laboratory findings were typically seen in our patient with marked elevation of both glutaric acids as well as its different subtypes. The findings of CT scan brain were consistent with findings in literature.10,11

Enzymatic confirmation of the diagnosis of GA should ideally be done by measurement of GCDH enzyme activity or mutation analysis. It was, however, not carried out in this patient due to non-availability of the facility in our set-up. The other two types of GA (2 and 3) were presumptively excluded due to their extremely rare occurrence, and presentation at early infancy with lethal neuro-metabolic manifestations. ${ }^{12}$ The aim of treatment is to reduce urine glutaric acids and its subtype levels to as close to normal as possible while maintaining normal growth rate with low protein, low lysine, and carnitine supplementation in order to reduce acute encephalopathic events. All children presenting with neurological manifestations of metabolic disorder should be thoroughly worked up for diagnosis and subsequent early initiation of therapy for prevention of permanent disability.

\section{REFERENCES}

1. Kölker S, Christensen E, Leonard JV. Diagnosis and management of glutaric aciduria type I - revised recommendations; J Inherit Metab Dis 2011; 34:677-94.

2. Tsiotou AG. The child with glutaric aciduria type I: anesthetic and perioperative management; $J$ Anesth 2011; 25:301-4.

3. Afroze B, Lakhani L, Naz F, Somani S, Yunus Z, Brown N. Challenges identified in the management of patients with inherited metabolic disorders - A five-year experience from Pakistan. The Egyptian J Med Human Genetics 2016; 17: 259-64.

4. Afroze B, Yunus ZM. Glutaric acidura type-1 importance of early diagnosis and treatment. J Pak Med Assoc 2014:593-5.

5. Sherazi NA, Khan AH, Jafri L, Jamil A, Khan NA, Afroze B. Selective screening for organic acidurias and amino acidopathies in Pakistani children. J Coll Physicians Surg Pak 2017; 27:218-21.

6. Divry P, Vianey-Liaud C, Cotte J. Routine gas chromatographic/mass spectrometric analysis of urinary organic acids. Results over a three-year period. Biomed Environ Mass Spectrom 1987; 14:663-8.

7. Baric I, ZSchoke J, Christensen E, Duran M, Goodman SI. Diagnosis and manangement of glutaric aciduria type I. $J$ Inherit Metab Dis 1998; 21:326-40.

8. Kamate M, Patil V, Chetal V, Darak P, Hattiholi V. Glutaric aciduria type I: A treatable neurometabolic disorder. Ann Indian Acad Neurol 2012; 15:31-4.

9. Boy N, Mühlhausen C, Maier EM. Proposed recommendations for diagnosing and managing individuals with glutaric aciduria type I: second revision. J Inherit Metab Dis 2017; 40:75-101.

10. Gupta N, Kumar Singh P, Kumar M, Shastri S, Gulati S, Kumar A, et al. Glutaric acidemia type 1-clinico-molecular profile and novel mutations in GCDH gene in Indian patients. JIMD Rep 2015; 21:45-55.

11. Fraidakis MJ, Liadinioti C, Stefanis L, Dinopoulos A, Pons R, Papathanassiou M. Rare late-onset presentation of glutaric aciduria type I in a 16-year-old woman with a novel GCDH mutation. JIMD Rep 2015; 18:85-92.

12. Knerr I, Zschocke J, Trautmann U, Dorland L, de Koning TJ, Muller $\mathrm{P}$, et al. Glutaric aciduria type III: A distinctive nondisease? J Inherit Metab Dis 2002; 25:483-90.

......... 